\title{
Relaxation of the chiral imbalance in dense matter of a neutron star
}

\author{
Maxim Dvornikov ${ }^{1,2,3, \star}$ \\ ${ }^{1}$ Pushkov Institute of Terrestrial Magnetism, lonosphere and Radiowave Propagation (IZMIRAN), \\ Kaluzhskoe HWY 4, 142190 Troitsk, Moscow, Russia \\ ${ }^{2}$ Physics Faculty, National Research Tomsk State University, 36 Lenin Avenue, 634050 Tomsk, Russia \\ ${ }^{3}$ University of Hamburg, II Institute for Theoretical Physics, 149 Luruper Chaussee, \\ D-22761 Hamburg, Germany
}

\begin{abstract}
Using the quantum field theory methods, we calculate the helicity flip of an electron scattering off protons in dense matter of a neutron star. The influence of the electroweak interaction between electrons and background nucleons on the helicity flip is examined. We also derive the kinetic equation for the chiral imbalance. The derived kinetic equation is compared with the results obtained by other authors.
\end{abstract}

\section{Introduction}

Some neutron stars (NS) can possess extremely strong magnetic fields $B \gtrsim 10^{15} \mathrm{G}$. These NSs are called magnetars [1]. Despite long observational history of magnetars and numerous theoretical models for the generation of their magnetic fields, nowadays there is no commonly accepted mechanism explaining the origin of magnetic fields in these compact stars.

In Refs. [2-4] we developed the new model for the generation of magnetic fields in magnetars. In this model a large-scale magnetic field in NS can be amplified to the strenth predicted in magnetars owing to the magnetic field instability driven by the electron-nucleon $(e N)$ electroweak interaction. Using the proposed model we could account for some properties of magnetars, e.g., ages of these compact stars. Despite the plausibility of the model in Refs. [2-4], some of its ingredients should be substantiated by more detailed calculations based on reliable methods of the quantum field theory (QFT). This work is devoted to the further development of the proposed description of the magnetic fields generation in magnetars. In particular, we will be interested in the evolution of the chemical potentials of the electron gas in NS in the presence of background nucleons.

\section{Electron-proton collisions in dense plasma}

In NS, the helicity of a massive electron can be changed in electron-proton (ep) and electron-electron electromagnetic scatterings as well as in the interaction of an electron with the anomalous magnetic moment of a neutron. As found in Ref. [5], the rate of the former reaction in dense matter of NS is higher than that of the later ones. Therefore, in our analysis, we shall account for only ep collisions.

^e-mail: maxdvo@izmiran.ru 


\subsection{Helicity flip rate in $e p$ collisions}

The matrix element for the ep collision, due to the electromagnetic interaction, has the form,

$$
\mathcal{M}=\frac{\mathrm{i} e^{2}}{\left(k_{1}-k_{2}\right)^{2}} \bar{e}\left(p_{2}\right) \gamma^{\mu} e\left(p_{1}\right) \cdot \bar{p}\left(k_{2}\right) \gamma_{\mu} p\left(k_{1}\right),
$$

where $e>0$ is the absolute value of the electron charge, $\gamma^{\mu}=\left(\gamma^{0}, \gamma\right)$ are the Dirac matrices, $p_{1,2}^{\mu}=$ $\left(E_{1,2}, \mathbf{p}_{1,2}\right)$ and $k_{1,2}^{\mu}=\left(\mathcal{E}_{1,2}, \mathbf{k}_{1,2}\right)$ are the four momenta of electrons and protons. The momenta of incoming particles are marked with the label 1 and that of the outgoing particles with the label 2.

We shall consider a process when a proton is in the unpolarized states before and after the collision whereas an electron changes its polarization in the scattering. The square of the matrix element in Eq. (1) reads

$$
|\mathcal{M}|^{2}=\frac{e^{4}}{\left(k_{1}-k_{2}\right)^{4}} \operatorname{tr}\left[\rho_{p}\left(k_{1}\right) \gamma^{\mu} \rho_{p}\left(k_{2}\right) \gamma^{\nu}\right] \cdot \operatorname{tr}\left[\rho_{e}\left(p_{1}\right) \gamma_{\mu} \rho_{e}\left(p_{2}\right) \gamma_{\nu}\right]
$$

where the spin density matrices of protons $\rho_{p}\left(k_{1,2}\right)$ and electrons $\rho_{e}\left(p_{1,2}\right)$ are [6, pp. 106-111]

$$
\rho_{p}\left(k_{1,2}\right)=\frac{1}{2}\left(\gamma_{\mu} k_{1,2}^{\mu}+M\right), \quad \rho_{e}\left(p_{1,2}\right)=\frac{1}{2}\left(\gamma_{\mu} p_{1,2}^{\mu}+m\right)\left(1+\gamma^{5} \gamma_{\mu} a_{1,2}^{\mu}\right),
$$

where $M$ and $m$ are the masses of a proton and an electron, and $\gamma^{5}=\mathrm{i} \gamma^{0} \gamma^{1} \gamma^{2} \gamma^{3}$. The polarization vector of an electron $a^{\mu}$ in Eq. (3) has the form,

$$
a^{\mu}=\left(\frac{(\boldsymbol{\zeta} \cdot \mathbf{p})}{m}, \boldsymbol{\zeta}+\frac{\mathbf{p}(\boldsymbol{\zeta} \cdot \mathbf{p})}{m(E+m)}\right) .
$$

where $\zeta$ is the invariant three vector of the particle polarization, which describes the polarization in the particle rest frame. We shall suppose that electrons are in pure spin states with $\zeta^{2}=1$.

Let us fix the polarizations of an electron. If we study the $R \rightarrow L$ transition, i.e. we take that $\zeta_{1}=\mathbf{n}_{1}$ and $\zeta_{2}=-\mathbf{n}_{2}$, one gets that $a_{1,2}^{\mu}=\left( \pm p_{1,2}, \pm E_{1,2} \mathbf{n}_{1,2}\right) / m$, where $\mathbf{n}_{1,2}=\mathbf{p}_{1,2} / p_{1,2}$ are the unit vectors towards the electron momenta and $p_{1,2}=\left|\mathbf{p}_{1,2}\right|$. Using the approximation of the elastic ep scattering, i.e. assuming that $E_{1}=E_{2}$, and keeping only the leading term in the electron mass $m$ in the computation of the traces of the Dirac matrices in Eq. (2), one gets the matrix element squared in the form,

$$
|\mathcal{M}|^{2}=2 e^{4} m^{2}\left[1-\left(\mathbf{n}_{1} \cdot \mathbf{n}_{2}\right)\right] \frac{\left[\mathcal{E}_{1} \mathcal{E}_{2}+M^{2}+\left(\mathbf{k}_{1} \cdot \mathbf{k}_{2}\right)\right]}{\left[\left(\mathcal{E}_{1}-\mathcal{E}_{2}\right)^{2}-\left(\mathbf{k}_{1}-\mathbf{k}_{2}\right)^{2}\right]^{2}},
$$

which will be used in the following calculations.

The total probability of the ep collision in the ep plasma has the form [6, pp. 247-252],

$$
\begin{aligned}
W= & \frac{V}{8(2 \pi)^{8}} \int \frac{\mathrm{d}^{3} p_{1} \mathrm{~d}^{3} p_{2} \mathrm{~d}^{3} k_{1} \mathrm{~d}^{3} k_{2}}{E_{1} E_{2} \mathcal{E}_{1} \mathcal{E}_{2}} \delta^{4}\left(p_{1}+k_{1}-p_{2}-k_{2}\right)|\mathcal{M}|^{2} \\
& \times f_{e}\left(E_{1}-\mu_{\mathrm{R}}\right)\left[1-f_{e}\left(E_{2}-\mu_{\mathrm{L}}\right)\right] f_{p}\left(\mathcal{E}_{1}-\mu_{p}\right)\left[1-f_{p}\left(\mathcal{E}_{2}-\mu_{p}\right)\right],
\end{aligned}
$$

where we summed over the polarizations of the outgoing proton. Here $f_{e, p}(E)=[\exp (\beta E)+1]^{-1}$ are the Fermi-Dirac distributions of electrons and protons, $\mu_{p}$ is the chemical potential of protons, and $V$ is the normalization volume. In Eq. (6) we assume that incoming and outgoing electrons have 
different chemical potentials: $\mu_{\mathrm{R}}$ and $\mu_{\mathrm{L}}$ respectively. Protons and electrons are taken to be in the thermal equilibrium having the same temperature $T$.

Assuming that electrons are highly degenerate and ultrarelativistic, we can calculate the integrals over the electron momenta,

$$
I_{e}=\int \frac{\mathrm{d}^{3} p_{1} \mathrm{~d}^{3} p_{2}}{2 E_{1} 2 E_{2}} \frac{\left(p_{1} \cdot p_{2}\right)}{E_{1} E_{2}} \delta^{4}\left(p_{1}-p_{2}-q\right) \theta\left(\mu_{\mathrm{R}}-E_{1}\right) \theta\left(E_{2}-\mu_{\mathrm{L}}\right),
$$

where $q^{\mu}=k_{2}^{\mu}-k_{1}^{\mu}$ and and $\left(p_{1} \cdot p_{2}\right)=E_{1} E_{2}\left[1-\left(\mathbf{n}_{1} \cdot \mathbf{n}_{2}\right)\right]$. Using the identity

$$
\int \frac{\mathrm{d}^{3} p}{2 E}=\int \mathrm{d}^{4} p \theta\left(p_{0}\right) \delta\left(p^{2}\right)
$$

and integrating over $p_{2}$ we can obtain that

$$
I_{e}=-\int \frac{\mathrm{d}^{3} p}{2 E} \frac{E q_{0}-(\mathbf{p} \cdot \mathbf{q})}{E\left(E-q_{0}\right)} \delta\left(2 E q_{0}-2(\mathbf{p} \cdot \mathbf{q})-q^{2}\right) \theta\left(\mu_{\mathrm{R}}-E\right) \theta\left(E-q_{0}-\mu_{\mathrm{L}}\right) .
$$

Here we recall that we use the approximation of the elastic scattering, i.e. $E_{1}=E_{2}$. Hence we should set $q_{0}=\mathcal{E}_{2}-\mathcal{E}_{1}=0$ in Eq. (9). Then we represent $\mathrm{d}^{3} p=2 \pi \mathrm{d} \cos \theta E^{2} \mathrm{~d} E$ and integrate over $\cos \theta$ using the remaining delta function

$$
\delta\left(2 E|\mathbf{q}| \cos \theta-\mathbf{q}^{2}\right)=\frac{1}{2 E|\mathbf{q}|} \delta\left(\cos \theta-\frac{|\mathbf{q}|}{2 E}\right) .
$$

Finally, we obtain

$$
I_{e}=\frac{\pi|\mathbf{q}|}{4} \int_{0}^{\infty} \frac{\mathrm{d} E}{E^{2}} \theta\left(\mu_{\mathrm{R}}-E\right) \theta\left(E-\mu_{\mathrm{L}}\right) \approx \frac{\pi|\mathbf{q}|}{4} \frac{\mu_{\mathrm{R}}-\mu_{\mathrm{L}}}{\mu_{e}^{2}},
$$

where we take that $\mu_{e} \approx \mu_{\mathrm{R}} \approx \mu_{\mathrm{L}}$ is the mean chemical potential of the electron gas. Moreover we assume that $\mu_{\mathrm{R}}>\mu_{\mathrm{L}}$ for the total probability to be positive.

Using Eqs. (5) and (11), one gets that the total probability in Eq. (6) takes the form,

$$
\begin{aligned}
W= & \frac{V \pi e^{4} m^{2}}{4(2 \pi)^{8}} \frac{\mu_{\mathrm{R}}-\mu_{\mathrm{L}}}{\mu_{e}^{2}} \int \frac{\mathrm{d}^{3} k_{1} \mathrm{~d}^{3} k_{2}}{\mathcal{E}_{1} \mathcal{E}_{2}} \frac{\mathcal{E}_{1} \mathcal{E}_{2}+M^{2}+\left(\mathbf{k}_{1} \cdot \mathbf{k}_{2}\right)}{\left[\left(\mathbf{k}_{1}-\mathbf{k}_{2}\right)^{2}+\omega_{p}^{2}\right]^{3 / 2}} \\
& \times f_{p}\left(\mathcal{E}_{1}-\mu_{p}\right)\left[1-f_{p}\left(\mathcal{E}_{2}-\mu_{p}\right)\right],
\end{aligned}
$$

Here, in the denominator, we replace $\left(\mathbf{k}_{1}-\mathbf{k}_{2}\right)^{2} \rightarrow\left(\mathbf{k}_{1}-\mathbf{k}_{2}\right)^{2}+\omega_{p}^{2}$, where $\omega_{p}$ is the plasma frequency in the $e p$ plasma, to avoid the infrared divergencies. Now one can compute the integrals over the proton momenta,

$$
\begin{aligned}
I_{p}= & \int \frac{\mathrm{d}^{3} k_{1} \mathrm{~d}^{3} k_{2}}{\mathcal{E}_{1} \mathcal{E}_{2}} \frac{\mathcal{E}_{1} \mathcal{E}_{2}+M^{2}+\left(\mathbf{k}_{1} \cdot \mathbf{k}_{2}\right)}{\left[\left(\mathbf{k}_{1}-\mathbf{k}_{2}\right)^{2}+\omega_{p}^{2}\right]^{3 / 2}} f_{p}\left(\mathcal{E}_{1}-\mu_{p}\right)\left[1-f_{p}\left(\mathcal{E}_{2}-\mu_{p}\right)\right] \\
& \approx 32 \sqrt{2 \tilde{\mu}_{p}} M^{3 / 2} \pi^{2} T J
\end{aligned}
$$

where

$$
J=\int_{0}^{1} \mathrm{~d} x \frac{x^{2} \sqrt{1-x^{2}}}{\left(x^{2}+L^{-2}\right)^{3 / 2}}=\frac{1}{\sqrt{1+L^{2}}} \boldsymbol{K}\left(\frac{L}{\sqrt{1+L^{2}}}\right)\left[L+\frac{2}{L}\right]-\frac{2}{L} \boldsymbol{E}\left(\frac{L}{\sqrt{1+L^{2}}}\right) \sqrt{1+L^{2}} .
$$


Here $L^{2}=8 M \tilde{\mu}_{p} / \omega_{p}^{2}, \boldsymbol{K}(z)$ and $\boldsymbol{E}(z)$ are the complete elliptic integrals, and $\tilde{\mu}_{p}=\mu_{p}-M$ is the nonrelativistic part of the protons chemical potential. To derive Eq. (13) we assume that protons are nonrelativistic and the scattering is elastic. Moreover, the proton gas is supposed to have a small nonzero temperature.

Assuming that in a degenerate plasma one has $\omega_{p}^{2}=4 \alpha_{\mathrm{em}} \mu_{e}^{2} / 3 \pi$ (see, e.g., Ref. [7]), where $\alpha_{\mathrm{em}}=$ $e^{2} / 4 \pi$ is the fine structure constant, and $\tilde{\mu}_{p} \approx \mu_{e}^{2} / 2 M$ due to the electroneutrality of the NS matter, we get that $L^{2}=3 \pi / \alpha_{\mathrm{em}} \approx 1291 \gg 1$. Decomposing $J$ in Eq. (14) in this limit, one obtains

$$
J=\ln 4 L-2+O\left(L^{-1}\right)=\frac{1}{2}\left[\ln \left(\frac{48 \pi}{\alpha_{\mathrm{em}}}\right)-4\right]>0 .
$$

Finally, using Eqs. (12)-(15), we get the total probability for the helicity flip in an ep collision,

$$
W=W_{0}\left(\mu_{\mathrm{R}}-\mu_{\mathrm{L}}\right) \theta\left(\mu_{\mathrm{R}}-\mu_{\mathrm{L}}\right), \quad W_{0}=\frac{V e^{4}}{32 \pi^{5}} \frac{m^{2} M}{\mu_{e}} T\left[\ln \left(16 L^{2}\right)-4\right] .
$$

Note that the total probability is always positive. That is why we introduce the step function $\theta\left(\mu_{\mathrm{R}}-\mu_{\mathrm{L}}\right)$ in Eq. (16). In the next section we shall compare Eq. (16) with the analogous expression accounting for the electroweak interaction between electrons and neutrons.

\subsection{Helicity flip rate of electrons, electroweakly interacting with nuclear matter}

If we take into account the electroweak interaction between electrons and nucleons, the matrix element for the $e p$ scattering has the same form as in Eq. (1). However, instead of the electron spinors in vacuum, we should use the exact solutions of the Dirac equation for an electron, interacting with a background matter, found in Appendix A.

We shall start with the analysis of $R \rightarrow L$ transitions. According to Eq. (1) it is necessary to compute the following quantity:

$$
J^{\mu}=\left(J_{0}, \boldsymbol{J}\right)=\bar{u}_{-}\left(p_{2}\right) \gamma^{\mu} u_{+}\left(p_{1}\right) .
$$

Using Eqs. (31) and (33) one gets

$$
\begin{aligned}
J_{0} & =-\frac{m P_{0}\left[p_{1}+p_{2}+E_{+}\left(p_{1}\right)+E_{-}\left(p_{2}\right)-2 \bar{V}\right]}{2 \sqrt{E_{0+}\left(p_{1}\right) E_{0-}\left(p_{2}\right)\left[E_{-}\left(p_{2}\right)+p_{2}-V_{\mathrm{R}}\right]\left[E_{+}\left(p_{1}\right)+p_{1}-V_{\mathrm{L}}\right]}}, \\
\boldsymbol{J} & =-\frac{m \mathbf{P}\left[p_{1}-p_{2}+E_{+}\left(p_{1}\right)-E_{-}\left(p_{2}\right)-2 V_{5}\right]}{2 \sqrt{E_{0+}\left(p_{1}\right) E_{0-}\left(p_{2}\right)\left[E_{-}\left(p_{2}\right)+p_{2}-V_{\mathrm{R}}\right]\left[E_{+}\left(p_{1}\right)+p_{1}-V_{\mathrm{L}}\right]}},
\end{aligned}
$$

where

$$
P_{0}=w_{-}^{\dagger}\left(\mathbf{p}_{2}\right) w_{+}\left(\mathbf{p}_{1}\right), \quad \mathbf{P}=w_{-}^{\dagger}\left(\mathbf{p}_{2}\right) \boldsymbol{\sigma} w_{+}\left(\mathbf{p}_{1}\right) .
$$

Here $w_{ \pm}$are the two component spinors corresponding to different helicities, which are defined in Ref. [6, p. 86], $\sigma$ are the Pauli matrices, $V_{\mathrm{L}, \mathrm{R}}$ are the effective potentials of the interaction of left and right chiral projections of an electron field with the background matter, defined in Eq. (29), $\bar{V}=$ $\left(V_{\mathrm{L}}+V_{\mathrm{R}}\right) / 2$, and $V_{5}=\left(V_{\mathrm{L}}-V_{\mathrm{R}}\right) / 2$. To obtain Eq. (18) we use the Dirac matrices in the chiral representation [8, pp. 691-696].

For ultrarelativistic electrons one obtains from Eq. (30) that $E_{ \pm}\left(p_{1,2}\right)=p_{1,2}+V_{\mathrm{R}, \mathrm{L}}$. As in Sec. 2.1, here we also study the elastic scattering, in which colliding particles do not transfer to exited states, i.e. $E_{+}\left(p_{1}\right)=E_{-}\left(p_{2}\right)$ and $\mathcal{E}_{1}=\mathcal{E}_{2}$. The equality of energies of incoming and outgoing ultrarelativistic 
electrons is equivalent to $p_{1}-p_{2}=2 V_{5}$. Thus, one obtains that $\boldsymbol{J}=0$. The computation of $P_{0}$ in Eq. (19) gives $\left|P_{0}\right|^{2}=\left[1-\left(\mathbf{n}_{1} \cdot \mathbf{n}_{2}\right)\right] / 2$. Thus, using Eq. (18), one gets that the square of matrix element in Eq. (1) has the form,

$$
|\mathcal{M}|^{2}=e^{4} m^{2} \frac{\left(p_{1}+p_{2}\right)^{2}\left[1-\left(\mathbf{n}_{1} \cdot \mathbf{n}_{2}\right)\right]}{8\left(p_{1}-V_{5}\right)^{2}\left(p_{2}+V_{5}\right)^{2}} \frac{\left[\mathcal{E}_{1} \mathcal{E}_{2}+M^{2}+\left(\mathbf{k}_{1} \cdot \mathbf{k}_{2}\right)\right]}{\left[\left(\mathcal{E}_{1}-\mathcal{E}_{2}\right)^{2}-\left(\mathbf{k}_{1}-\mathbf{k}_{2}\right)^{2}\right]^{2}},
$$

where we keep only the leading term in the electron mass.

The total probability of the process has the form,

$$
\begin{aligned}
W= & \frac{V}{2(2 \pi)^{8}} \int \frac{\mathrm{d}^{3} p_{1} \mathrm{~d}^{3} p_{2} \mathrm{~d}^{3} k_{1} \mathrm{~d}^{3} k_{2}}{\mathcal{E}_{1} \mathcal{E}_{2}} \delta^{4}\left(p_{1}+k_{1}-p_{2}-k_{2}\right)|\mathcal{M}|^{2} \\
& \times f_{e}\left(E_{1}-\mu_{\mathrm{R}}\right)\left[1-f_{e}\left(E_{2}-\mu_{\mathrm{L}}\right)\right] f_{p}\left(\mathcal{E}_{1}-\mu_{p}\right)\left[1-f_{p}\left(\mathcal{E}_{2}-\mu_{p}\right)\right] .
\end{aligned}
$$

Note that Eq. (21) is slightly different from Eq. (6). It can be accounted for by the different way to normalize electron basis spinors; cf. Eq. (32).

As in Sec. 2.1, first, in Eq. (21) we compute the integrals over the electron momenta,

$$
\begin{aligned}
I_{e}= & \int \frac{\mathrm{d}^{3} p_{1} \mathrm{~d}^{3} p_{2}\left(p_{1}+p_{2}\right)^{2}\left[1-\left(\mathbf{n}_{1} \cdot \mathbf{n}_{2}\right)\right]}{16\left(p_{1}-V_{5}\right)^{2}\left(p_{2}+V_{5}\right)^{2}} \\
& \times \delta^{4}\left(p_{1}-p_{2}-q\right) \theta\left(\mu_{\mathrm{R}}-p_{1}-V_{\mathrm{R}}\right) \theta\left(p_{2}+V_{\mathrm{L}}-\mu_{\mathrm{L}}\right),
\end{aligned}
$$

where we suppose that incoming and outgoing electrons are ultrarelativistic, highly degenerate, and have different chemical potentials. We remind that we study $R \rightarrow L$ transitions. After the integration over $\mathbf{p}_{2}$ with help of the delta function, one obtains that

$$
\begin{aligned}
I_{e}= & \int \frac{\mathrm{d}^{3} p(p+|\mathbf{p}-\mathbf{q}|)^{2}}{16\left(p-V_{5}\right)^{2}\left(|\mathbf{p}-\mathbf{q}|+V_{5}\right)^{2}}\left[1-\frac{(\mathbf{p} \cdot \mathbf{p}-\mathbf{q})}{p|\mathbf{p}-\mathbf{q}|}\right] \\
& \times \delta\left(p-2 V_{5}-|\mathbf{p}-\mathbf{q}|\right) \theta\left(\mu_{\mathrm{R}}-p-V_{\mathrm{R}}\right) \theta\left(|\mathbf{p}-\mathbf{q}|+V_{\mathrm{L}}-\mu_{\mathrm{L}}\right) .
\end{aligned}
$$

Then we represent $\mathrm{d}^{3} p=2 \pi \mathrm{d} \cos \theta p^{2} \mathrm{~d} p$ and integrate over $\cos \theta$ using the remaining delta function

$$
\delta\left(p-2 V_{5}-|\mathbf{p}-\mathbf{q}|\right)=\frac{p-2 V_{5}}{p|\mathbf{q}|} \delta\left(\cos \theta-\frac{|\mathbf{q}|^{2}+4 p V_{5}-V_{5}^{2}}{2 p|\mathbf{q}|}\right) .
$$

Finally we get the following expression for $I_{e}$ :

$$
\begin{aligned}
I_{e}= & \pi \frac{|\mathbf{q}|^{2}-V_{5}^{2}}{4|\mathbf{q}|} \int_{\mu_{\mathrm{L}}-V_{\mathrm{R}}}^{\mu_{\mathrm{R}}-V_{\mathrm{R}}} \frac{\mathrm{d} p}{\left(p-V_{5}\right)^{2}}=\pi \frac{|\mathbf{q}|^{2}-V_{5}^{2}}{4|\mathbf{q}|} \frac{\left(\mu_{\mathrm{R}}-\mu_{\mathrm{L}}\right)}{\left(\mu_{\mathrm{R}}-\bar{V}\right)\left(\mu_{\mathrm{L}}-\bar{V}\right)} \\
& \approx \frac{\pi|\mathbf{q}|}{4} \frac{\left(\mu_{\mathrm{R}}-\mu_{\mathrm{L}}\right)}{\mu_{e}^{2}}
\end{aligned}
$$

where we suppose that $\mu_{\mathrm{R}, \mathrm{L}} \gg \bar{V}, \mu_{\mathrm{R}} \approx \mu_{\mathrm{L}} \approx \mu_{e}$, and $|\mathbf{q}| \gg V_{5}$. The most important consequence of Eq. (25) consists in the fact that the $e N$ interaction does not contribute to the difference of the chemical potentials in the numerator.

Eventually, on the basis of Eqs. (21) and (25), one gets the expression for the total probability which coincides with that in Eq. (12). Performing the integration over the protons momenta as in 
Sec. 2.1, we obtain that the total probability for the helicity flip in an $e p$ collision has the same form as in Eq. (16). If we study $L \rightarrow R$ transitions, making analogous calculations as in the $R \rightarrow L$ case, we derive the expression for $W$, which also coincides with that in Eq. (16). For the sake of brevity we omit these computations.

At the end of this section we shall analyze the approximations made to derive the total probability in the presence of the electroweak $e N$ interaction. First, we assume that $e p$ collisions are elastic. As found in Ref. [9, pp. 205-208], the inelastic contributions to the relativistic collision integral in case of the scattering due to the long range Coulomb interaction are suppressed compared to elastic ones. This situation takes place in our work.

The differential probability of the helicity flip (the analogue of the differential cross section) is $\mathrm{d} W \sim \mathrm{d} \chi / \chi$, where $\chi$ is the scattering angle. The maximal probability corresponds to $\chi \sim 0$. That is why we introduce $\omega_{p} \neq 0$ in Eq. (12) to regularize this infrared divergence. One can estimate $\chi_{\text {min }}$ using Eq. $(15)$ as $\chi_{\text {min }} \sim \alpha_{\text {em }} / 48 \pi=4.8 \times 10^{-5}$. Note that the Coulomb cut-off parameter $L_{\mathrm{C}}=\ln \left(\chi_{\min }^{-1}\right) \sim 10$ is typical for relativistic plasmas. The energy of an electron can change by $\Delta E=\left|E_{1}-E_{2}\right| \sim \chi^{2}\langle E\rangle$ in a collision, where $\langle E\rangle \sim 10^{2} \mathrm{MeV}$ is the mean electron energy. If we define $\chi_{5}$ corresponding to $\Delta E \sim 2 V_{5}$, i.e. when the inelastic effects become comparable with the electroweak interaction contribution, it reads, $\chi_{5}=3.2 \times 10^{-4}$. Thus, one gets that $\chi_{5} \gg \chi_{\min }$, i.e. there will be many particles with a big contribution to $\mathrm{d} W$, for which the energy change in a collision is less than $2 V_{5}$. Hence the elasticity is a good approximation even in the presence of the $e N$ interaction. Moreover, the approximation of the elastic ep scattering was also used in Ref. [10], where $\Gamma_{f}$ was computed in the situation when protons are nondegenerate. If inelastic effects are taken into account, it can result in some dependence of $W_{0}$ in Eq. (16) on $V_{\mathrm{L}, \mathrm{R}}$. However, $V_{5}$ still will not contribute to the factor $\mu_{\mathrm{R}}-\mu_{\mathrm{L}}$ in Eq. (16).

Second, the proton energies in Eq. (22) are taken as in vacuum: $\mathcal{E}_{1,2}=\sqrt{k_{1,2}^{2}+M^{2}}$, whereas for electrons we exactly account for the contribution of the electroweak interaction to the electron wave function in Eqs. (17) and (18) and and to the electron energies in Eq. (23). However, protons like electrons can electroweakly interact with background neutrons. To justify our choice of the proton energies, we define the analogues of $V_{\mathrm{L}, \mathrm{R}}$ for protons and denote them as $V_{\mathrm{L}, \mathrm{R}}^{(p)}$. Using Eq. (30), we can estimate the contribution of the electroweak $p N$ interaction to the proton energies as $\left|\Delta\left(\mathcal{E}_{1,2}\right)_{\mathrm{EW}}\right| \sim$ $\bar{V}_{p} \mp k_{1,2} V_{5}^{(p)} / M$, where $\bar{V}_{p}=\left[V_{\mathrm{L}}^{(p)}+V_{\mathrm{R}}^{(p)}\right] / 2$ and $V_{5}^{(p)}=\left[V_{\mathrm{L}}^{(p)}-V_{\mathrm{R}}^{(p)}\right] / 2$. Now, in the energy conservation delta function in Eq. (22), one has $\left|\Delta\left(\mathcal{E}_{1}-\mathcal{E}_{2}\right)_{\mathrm{EW}}\right| \lesssim p_{\mathrm{F}_{\mathrm{p}}} V_{5}^{(p)} / M \sim 0.1 V_{5}$ since $p_{\mathrm{F}_{\mathrm{p}}} \sim 10^{2} \mathrm{MeV}$ is the Fermi momentum for protons in NS, $M \sim 1 \mathrm{GeV}$, and $V_{5}^{(p)} \sim V_{5}$. Analogously for electrons we have $\left|\Delta\left(E_{1}-E_{2}\right)_{\mathrm{EW}}\right|=2 V_{5}$. Thus the contribution of the electroweak $p N$ interaction to the conservation of energy is negligible compared to that of the $e N$ interaction: $\left|\Delta\left(\mathcal{E}_{1}-\mathcal{E}_{2}\right)_{\mathrm{EW}}\right| \ll\left|\Delta\left(E_{1}-E_{2}\right)_{\mathrm{EW}}\right|$.

\subsection{Kinetics of the chiral imbalance}

Basing on Eq. (16), one gets the kinetic equations for the total numbers of right and left electrons $N_{\mathrm{R}, \mathrm{L}}$ as

$$
\begin{gathered}
\frac{\mathrm{d} N_{\mathrm{R}}}{\mathrm{d} t}=-W(R \rightarrow L)+W(L \rightarrow R)=-W_{0}\left(\mu_{\mathrm{R}}-\mu_{\mathrm{L}}\right), \\
\frac{\mathrm{d} N_{\mathrm{L}}}{\mathrm{d} t}=-W(L \rightarrow R)+W(R \rightarrow L)=-W_{0}\left(\mu_{\mathrm{L}}-\mu_{\mathrm{R}}\right),
\end{gathered}
$$

where $\mu_{\mathrm{R}, \mathrm{L}}$ are the chemical potentials of right and left electrons. Let us introduce the number densities as $n_{\mathrm{R}, \mathrm{L}}=N_{\mathrm{R}, \mathrm{L}} / V$. Using the standard relation between $n_{\mathrm{R}, \mathrm{L}}$ and $\mu_{\mathrm{R}, \mathrm{L}}[2]$, we get that $\mathrm{d}\left(n_{\mathrm{R}}-n_{\mathrm{L}}\right) / \mathrm{d} t \approx$ 
$2 \dot{\mu}_{5} \mu_{e}^{2} / \pi^{2}$, where $\mu_{5}=\left(\mu_{\mathrm{R}}-\mu_{\mathrm{L}}\right) / 2$ is the chiral imbalance. Finally, we get the kinetic equation for $\mu_{5}$,

$$
\frac{\mathrm{d} \mu_{5}}{\mathrm{~d} t}=-\Gamma_{f} \mu_{5}, \quad \Gamma_{f}=\frac{\alpha_{\mathrm{em}}^{2}}{\pi}\left[\ln \left(\frac{48 \pi}{\alpha_{\mathrm{em}}}\right)-4\right]\left(\frac{m}{\mu_{e}}\right)^{2}\left(\frac{M}{\mu_{e}}\right) T,
$$

where we use Eqs. (16) and (26).

One can see in Eq. (27) that the helicity flipping term contains $\mu_{5}$ rather than $\mu_{5}+V_{5}$ as recently suggested in Ref. [11]. Note that this our result is based on the explicit QFT calculation of the total probability of the $e p$ scattering, where we exactly account for the $e N$ electroweak interaction.

We also mention that, in Eq. (27), we corrected the value of $\Gamma_{f}$ used in Refs. [2-4]. The reason for the discrepancy of $\Gamma_{f}$ consists in the fact that in Ref. [2] we relied on the results of Ref. [5], where the scattering of unpolarized electrons off protons was studied. However, in our case it is essential to have the fixed opposite polarizations of incoming and outgoing electrons. Hence, the matrix elements used in the present work and in Ref. [5] are different. This fact, explains, e.g., that $\Gamma_{f}$ in Eq. (27) is linear in $T$ whereas that used in Refs. [2-4] is proportional to $T^{2}$.

We also mention that $\Gamma_{f}$ was recently calculated in Ref. [10]. The value of $\Gamma_{f}$ obtained in Ref. [10] is independent of $T$ since it was assumed that protons are nondegenerate. This assumption is valid when the early stages of the NS evolution are considered. In Refs. [2-4], we studied the magnetic field generation in a thermally relaxed NS at $t \gtrsim 10^{2} \mathrm{yr}$ after the onset of the supernova collapse. At this time, the proton component of the NS matter should be taken as degenerate. Note that $\Gamma_{f} \sim \alpha_{\mathrm{em}}^{2}$ in Eq. (27) as in Ref. [10].

\section{Discussion}

The detailed computation of the helicity flip rate in ep collisions was the last missing ingredient of the new model, initially proposed in Ref. [2], for the generation of magnetic fields in magnetars driven by the parity violating $e N$ electroweak interaction. Note that, in Refs. [2,3], we estimated the helicity flip rate $\Gamma_{f}$ basing on the classical plasma physics. However, the particle spin is a purely quantum object and its evolution should be treated appropriately. In the present work we used the QFT methods to compute the helicity flip rate. It can explain the discrepancy of our results from those in Refs. [2, 3].

The second important result obtained in the present work was the analysis of the influence of the electroweak interaction of colliding electrons with background nucleons on the helicity flip process. Using the approximation of the elastic scattering and assuming that electrons are ultrarelativistic, we have found that the kinetic equation for the chiral imbalance should have the same form as in Refs. [2, 3], see Eq. (27), contrary to the claim in Ref. [11]. It should be also noted that the computation of the anomalous current along the magnetic field made in Refs. [2,3] and the calculation of the electron helicity flip in the present work imply the same definition of the chemical potentials $\mu_{\mathrm{L}, \mathrm{R}}$; see, e.g., the distribution functions of left and right electrons in Eq. (21)-(23) and in Eq. (7) in Ref. [2]. Thus the results of the present work are consistent with Refs. [2-4].

I am thankful to the organizers of Quarks 2016 for the inivitation, to the Tomsk State University Competitiveness Improvement Program, as well as to RFBR (research project No. 15-02-00293) and DAAD (grant No. 91610946) for partial support.

\section{A Solution of the Dirac equation for an electron, electroweakly interacting with nuclear matter}

Let us consider the electroneutral matter in NS consisting of neutrons, protons, and electrons. This matter is supposed to be at rest and unpolarized. The Dirac equation for a test electron, described by 
the bispinor wave function $\psi$, electroweakly interacting with neutrons and protons, has the form,

$$
\left[\mathrm{i} \gamma^{\mu} \partial_{\mu}-m-\gamma^{0}\left(V_{\mathrm{L}} P_{\mathrm{L}}+V_{\mathrm{R}} P_{\mathrm{R}}\right)\right] \psi=0,
$$

where

$$
V_{\mathrm{L}}=\frac{G_{\mathrm{F}}}{\sqrt{2}}\left[n_{n}-n_{p}(1-4 \xi)\right](1-2 \xi), \quad V_{\mathrm{R}}=-\frac{G_{\mathrm{F}}}{\sqrt{2}}\left[n_{n}-n_{p}(1-4 \xi)\right] 2 \xi,
$$

are the effective potentials of the interaction of left and right chiral projections, $n_{n, p}$ are the constant and uniform densities of neutrons and protons, $\xi=\sin ^{2} \theta_{\mathrm{W}} \approx 0.23$ is the Weinberg parameter, and $P_{\mathrm{L}, \mathrm{R}}=\left(1 \mp \gamma^{5}\right) / 2$ are the chiral projectors.

We shall look for the solution of Eq. (28) in the form, $\psi=e^{-\mathrm{i} E t+\mathrm{ipr}} u / \sqrt{V}$, where $u$ is the basis spinor. The energy levels of an electron have the form,

$$
E=\bar{V}+E_{0}, \quad \bar{V}=\frac{V_{\mathrm{L}}+V_{\mathrm{R}}}{2}, \quad E_{0}^{2}=\left(p-s V_{5}\right)^{2}+m^{2}, \quad V_{5}=\frac{V_{\mathrm{L}}-V_{\mathrm{R}}}{2},
$$

where $s= \pm 1$. If electrons are ultrarelativistic, one gets from Eq. (30) that $E_{ \pm}=p+V_{\mathrm{R}, \mathrm{L}}$. Note that the energy levels in Eq. (30) coincide with those found in Ref. [12].

The basis spinors $u_{ \pm}$for different helicities can be also obtained in the explicit form,

$$
u_{+}=N_{+}\left(\begin{array}{c}
w_{+} \\
-\frac{m}{E_{+}+p-V_{\mathrm{L}}} w_{+}
\end{array}\right), \quad u_{-}=N_{-}\left(\begin{array}{c}
-\frac{m}{E_{-}+p-V_{\mathrm{R}}} w_{-} \\
w_{-}
\end{array}\right),
$$

where $w_{ \pm}$are the basis two component spinors which are given in Ref. [6, p. 86]. The spinors $w_{ \pm}$are the eigenvectors of the helicity operator: $(\boldsymbol{\sigma} \cdot \mathbf{p}) w_{ \pm}= \pm|\mathbf{p}| w_{ \pm}$. Eq. (31) implies the chiral representation for the Dirac matrices [8, pp. 691-696].

If we normalize the electron wave function as

$$
\int \mathrm{d}^{3} x \psi^{\dagger} \psi=1
$$

we can find the normalization constants $N_{ \pm}$in Eq. (31) in the form,

$$
N_{ \pm}=\sqrt{\frac{E_{ \pm}+p-V_{\mathrm{L}, \mathrm{R}}}{2 E_{0 \pm}}},
$$

where $E_{ \pm}$and $E_{0 \pm}$ are given in Eq. (30).

\section{References}

[1] R. Turolla, S. Zane, and A. L. Watts, Rep. Prog. Phys. 78, 116901 (2015).

[2] M. Dvornikov and V. B. Semikoz, Phys. Rev. D 91, 061301 (2015).

[3] M. Dvornikov and V. B. Semikoz, J. Cosmol. Astropart. Phys. 05 (2015) 032.

[4] M. Dvornikov and V. B. Semikoz, Phys. Rev. D 92, 083007 (2015).

[5] D. C. Kelly, Astrophys. J. 179, 599 (1973).

[6] V. B. Berestetskii, E. M. Lifschitz, and L. P. Pitaevskii, Quantum Electrodynamics, 2nd ed. (Pergamon, Oxford, 1982).

[7] E. Braaten and D. Segel, Phys. Rev. D 48, 1478 (1993).

[8] C. Itzykson and J.-B. Zuber, Quantum field theory (McGraw-Hill, New York, 1980).

[9] E. M. Lifschitz and L. P. Pitaevskii, Physical Kinetics (Pergamon, Oxford, 1981).

[10] D. Grabowska, D. B. Kaplan, and S. Reddy, Phys. Rev. D 92, 085035 (2015).

[11] G. Sigl and N. Leite, J. Cosmol. Astropart. Phys. 01 (2016) 025.

[12] A. Grigoriev, et al., Grav. Cosmol. 14, 248 (2008). 